\title{
Draft genome sequence of Streptomyces tunisialbus DSM $105760^{\top}$
}

\author{
Ameni Ayed $^{1} \cdot$ Daniel Wibberg $^{2} \cdot$ Imène Zendah el Euch $^{3} \cdot$ Marcel Frese $^{3} \cdot$ Ferid Limam $^{1} \cdot$ Norbert Sewald $^{3}(0)$
}

Received: 28 October 2019 / Revised: 27 April 2020 / Accepted: 14 May 2020 / Published online: 30 May 2020

(c) The Author(s) 2020

\begin{abstract}
Streptomyces strains are well known as promising source of bioactive secondary metabolites, important in ecology, biotechnology and medicine. In this study, we present the draft genome of the new type strain Streptomyces tunisialbus DSM $105760^{\mathrm{T}}\left(=\mathrm{JCM} 32165^{\mathrm{T}}\right)$, a rhizospheric bacterium with antimicrobial activity. The genome is $6,880,753 \mathrm{bp}$ in size (average GC content, $71.85 \%$ ) and encodes 5802 protein-coding genes. Preliminary analysis with antiSMASH 5.1.2. reveals 34 predicted gene clusters for the synthesis of potential secondary metabolites, which was compared with those of Streptomyces varsoviensis NRRL ISP-5346.
\end{abstract}

Keywords Streptomyces tunisialbus $\cdot$ Draft genome $\cdot$ AntiSMASH 5.1.2 $\cdot$ Secondary metabolites

\section{Introduction}

Since the discovery of streptomycin in 1944 from Streptomyces griseus, the first antibiotic isolated from bacteria (Schatz et al. 1944), Actinobacteria have obtained large attention from the scientific community, mainly due to their ability in producing a wide spectrum of bioactive compounds with antimicrobial and antitumor activities (Demain 2014; Barka et al. 2016). Nowadays, this class provided more than $65 \%$ of antibiotics and antifungals used in medicine; including over 10,000 bioactive compounds that were produced by the members of the genus Streptomyces (Bérdy 2005, 2012; De Lima et al. 2012). This genus, proposed for the first time by Waksman and Henrici (1943), belong to the Gram-positive bacteria and is ubiquitous in nature (Kämpfer 2012). The classification of Streptomyces species is generally carried out using a polyphasic taxonomic approach, including chemotaxonomic, phenotypic, and genotypic characteristics

Communicated by Erko Stackebrandt.

Norbert Sewald

norbert.sewald@uni-bielefeld.de

1 Laboratory of Bioactive Substances, Biotechnology Center of Borj-Cedria (CBBC), BP-901, 2050 Hammam-Lif, Tunisia

2 Center for Biotechnology (CeBiTec), Bielefeld University, 33501 Bielefeld, Germany

3 Organic and Bioorganic Chemistry, Faculty of Chemistry, Bielefeld University, 33615 Bielefeld, Germany
(Anderson and Wellington 2001). Currently (Status April 2020), about 841 species of the genus Streptomyces with valid names have been described and published so far (https ://www.bacterio.net/streptomyces.html). Thus, several studies have been conducted to find new strains isolated from extreme habitats (Tiwari and Gupta 2012; Goodfellow 2013; Zhang et al. 2016). To cope with the major health problem of antibiotic-resistant microorganisms, many laboratories have focused on the discovery of new bioactive molecules produced from new species belonging to this genus. In fact, whole-genome sequencing (wgs) have opened new potential for better exploitation of useful secondary metabolites produced by this genus (Harrison and Studholme 2014; Lee et al. 2018; Ward and Allenb 2018). This fundamental technology was supported by the use of new bioinformatics tools like antiSMASH, which adds several new features, including prediction of gene cluster (Blin et al. 2017).

In this study, the genome of a new strain Streptomyces tunisialbus DSM $105760^{\mathrm{T}}$, which produces a broad spectrum of secondary metabolites with antibacterial and antifungal effects (Ayed et al. 2018), was sequenced. The genome sequence of S. tunisialbus was established to broaden the genomic basis for functional and comparative analyses focusing on secondary metabolite biosynthesis clusters of the strain. 


\section{Materials and methods}

The genomic DNA was extracted from a culture grown 3 days in ISP2 medium (Shirling and Gottlieb 1966), using cetyltrimethylammonium bromide (CTAB)-based method (Hopwood 2000). The quality of the DNA was assessed by gel-electrophoresis, and its quantity was determined by a fluorescence-based method using the Quant-iT PicoGreen dsDNA kit (Invitrogen) and the Tecan Infinite 200 Microplate Reader (Tecan Deutschland GmbH). For sequencing of the S. tunisialbus DSM $105760^{\mathrm{T}}$ genome, $4 \mu \mathrm{g}$ of purified DNA was used to generate a paired library (TruSeq DNA PCR-Free Library Prep Kit, Illumina). The draft genome sequence of strain $S$. tunisialbus was established by sequencing on an Illumina MiSeq system. To assemble the obtained and processed reads, the GS de novo Assembler software version 2.8 (Roche, Mannheim, Germany) with default setting was applied as recently described (Yücel et al. 2017). Annotation of the draft genome was performed by applying the GenDB 2.0 system (Meyer et al. 2003) and Prokka version 1.11 (Seemann 2014). To identify clusters involved in secondary metabolite biosynthesis, the annotated genome was analyzed by applying antiSMASH 5.1.2 (Blin et al. 2017). Based on the 16S analysis in Ayed et al., S. varsoviensis NRRL ISP-5346 was determined as closest relative with a sequenced genome. S. varsoviensis NRRL ISP-5346 was found to produce multiple hygrolide sub-families, e.g., hygrobafilomycins (JBIR-100 and hygrobafilomycin) and bafilomycins (bafilomycins C1 and D) (Molloy et al. 2016). Finally, comparative genome analysis for $S$. tunisialbus DSM $105760^{\mathrm{T}}$ and $S$. varsoviensis NRRL ISP5346 was performed by means of the comparative genomics tool EDGAR (Blom et al. 2009, 2016). To determine the relatedness between the $S$. tunisialbus DSM $105760^{\mathrm{T}}$ and $S$. varsoviensis NRRL ISP-5346 (Accession number: JOBF0100001-118), an average nucleotide identity analysis (ANI), average amino acid identity analysis (AAI) (Konstantinidis and Tiedje 2005) and a DDH estimation were performed as described previously (Meier-Kolthoff et al. 2013).

\section{Results and discussion}

The MiSeq sequencing run $(2 \times 300 \mathrm{bp})$ resulted in $2,092,356$ reads yielding approx. $627 \mathrm{Mb}$ sequence information for the sample representing on average a coverage of about 90 -fold. The final draft genome established by the GS Assembler software (version 2.8, Roche) consists of 35 contigs (N50: 455,831 bp) and 20 scaffolds (N50: 678,417 bp). The annotation of the draft genome of S. tunisialbus showed a linear chromosome which has a size of $6,880,753 \mathrm{bp}$ and encodes 5802 protein-coding sequences (CDS)s. The chromosome also contains 72 tRNA genes and eight rRNA operons (16S-23S-5S rRNA). The average GC content is $71.85 \%$ (Table 1).

An ANI value of $84.93 \%$, an AAI value of $87.43 \%$ and a DDH value of $25.00 \%$ were calculated for S. tunisialbus DSM $105760^{\mathrm{T}}$ and $S$. varsoviensis NRRL ISP-5346. High ANI, AAI values (above 95\%) and high DDH estimations (Formula 2 above 70\%) were usually observed for bacterial isolates representing the same species and are considered to indicate bacterial species demarcation previously (Konstantinidis and Tiedje 2005). Therefore, both strains are closely related, but do not belong to the same species.

By applying EDGAR, a comparative analysis was performed for S. tunisialbus DSM $105760^{\mathrm{T}}$ and S. varsoviensis NRRL ISP-5346. It appeared that 3830 genes corresponding to $66 \%$ and $55 \%$ of all genes identified in both draft genomes represent the core set of genes shared by both isolates. These core genes mainly encode for housekeeping functions, e.g., glycolysis. However, S. tunisialbus DSM $105760^{\mathrm{T}}$ possesses 1972 singletons, whereas 3062 singletons were identified for S. varsoviensis NRRL ISP-5346. Most of the S. tunisialbus DSM $105760^{\mathrm{T}}$ singletons were annotated as 'hypothetical' genes or transposases. However, genes encoding enzymes potentially involved in the synthesis of secondary metabolites were identified within the unique genes of $S$. tunisialbus DSM $105760^{\mathrm{T}}$. In addition, a few unique genes encoding for different cytochromes were found. For $S$. varsoviensis NRRL ISP-5346, the clusters found to produce hygrobafilomycins (JBIR-100 and hygrobafilomycin) and bafilomycins (bafilomycins $\mathrm{C} 1$ and $\mathrm{D}$ ) were unique.
Table 1 General features of $S$. tunisialbus DSM $105760^{\mathrm{T}}$ and S. varsoviensis NRRL ISP-5346

\begin{tabular}{lll}
\hline Features & S. tunisialbus DSM $105760^{\mathrm{T}}$ & S. varsoviensis NRRL ISP-5346 \\
\hline Length (bp) & $6,880,753$ & $8,588,807$ \\
Status & Draft genome (35 contigs) & Draft genome (118 contigs) \\
G+C content $(\%)$ & 71.85 & 72.40 \\
CDS & 5802 & 7229 \\
rRNAs operons & 8 & NA \\
tRNAs genes & 72 & 68 \\
\hline
\end{tabular}


Table 2 Secondary metabolite cluster of S. tunisialbus DSM $105760^{\mathrm{T}}$

\begin{tabular}{|c|c|c|c|}
\hline \multirow{2}{*}{$\frac{\text { Region }}{\text { Region } 1}$} & \multirow{2}{*}{$\begin{array}{l}\text { Type } \\
\text { NRPS-like,lassopeptide }\end{array}$} & \multicolumn{2}{|l|}{ Most similar known cluster } \\
\hline & & Citrulassin B & $100.00 \%$ \\
\hline Region 2 & Terpene & 2-Methylisoborneol & $100.00 \%$ \\
\hline Region 3 & T2PKS, T1PKS, NRPS, terpene & JBIR-76/JBIR-77 & $68.00 \%$ \\
\hline Region 4 & NRPS & C-1027 & $7.00 \%$ \\
\hline Region 5 & Terpene & Calicheamicin & $2.00 \%$ \\
\hline Region 6 & Ectoine & Ectoine & $100.00 \%$ \\
\hline Region 7 & Lanthipeptide & SapB & $75.00 \%$ \\
\hline Region 8 & Linaridin & Pentostatine/vidarabine & $12.00 \%$ \\
\hline Region 9 & Melanin & Melanin & $28.00 \%$ \\
\hline Region 10 & Melanin & Melanin & $28.00 \%$ \\
\hline Region 11 & Terpene & Aristeromycin & $6.00 \%$ \\
\hline Region 12 & Lassopeptide & Lagmysin & $60.00 \%$ \\
\hline Region 13 & T3PKS & Herboxidiene & $5.00 \%$ \\
\hline Region 14 & T3PKS, ladderane & Vazabitide A & $28.00 \%$ \\
\hline Region 15 & Butyrolactone & Griseoviridin/fijimycin A & $8.00 \%$ \\
\hline Region 16 & T1PKS & Elaiophylin & $8.00 \%$ \\
\hline Region 17 & T1PKS & Guadinomine & $7.00 \%$ \\
\hline Region 18 & T1PKS, thiopeptide, LAP, NRPS-like, terpene, NRPS & Aureothin & $100.00 \%$ \\
\hline Region 19 & hglE-KS & a201a & $5.00 \%$ \\
\hline Region 20 & Terpene & 2-Methylisoborneol & $75.00 \%$ \\
\hline Region 21 & hglE-KS, T3PKS & Alkylresorcinol & $100.00 \%$ \\
\hline Region 22 & NRPS, T1PKS, terpene & Althiomycin & $100.00 \%$ \\
\hline Region 23 & Bacteriocin, NRPS & Kirromycin & $16.00 \%$ \\
\hline Region 24 & T1PKS, Terpene, bacteriocin & Nystatin A1 & $63.00 \%$ \\
\hline Region 25 & NRPS & Deimino-antipain & $66.00 \%$ \\
\hline Region 26 & NRPS, terpene, T1PKS, transAT-PKS, PKS-like & Netropsin & $100.00 \%$ \\
\hline Region 27 & Siderophore & Vibrioferrin & $18.00 \%$ \\
\hline Region 28 & Terpene & Asukamycin & $4.00 \%$ \\
\hline Region 29 & Terpene & Hopene & $69.00 \%$ \\
\hline Region 30 & Indole & AT2433-A1 & $14.00 \%$ \\
\hline Region 31 & Butyrolactone & & \\
\hline Region 32 & NRPS & Bacillibactin & $46.00 \%$ \\
\hline Region 33 & T1PKS, NRPS, ladderane, arylpolyene, NRPS-like & Atratumycin & $55.00 \%$ \\
\hline
\end{tabular}

To identify all clusters involved in secondary metabolite biosynthesis, the $S$. tunisialbus DSM $105760^{\mathrm{T}}$ genome was analyzed by means of antiSMASH 5.1.2. The platform revealed the presence of 34 predicted gene clusters encode for potential secondary metabolites, such as antibiotics, antitumor compounds, fungicide, and lantibiotics. Likewise, six of these gene clusters are terpenes, such 2-methylisoborneol [100\%] a volatile organic compound well known for its biological activity, two for melanin biosynthesis and one for
Ectoine (Table 2). S. varsoviensis NRRL ISP-5346 has a similar portfolio of secondary metabolite clusters (Table 3).

The presence of a large number of cryptic secondary metabolite gene clusters in its genome, offers unsuspected potential for synthesizing bioactive compounds. In addition, our study shows that sequencing of new strains allows the discovery of new secondary metabolites relevant in ecology, biotechnology and medicine. 
Table 3 Secondary metabolite cluster of S. varsoviensis NRRL ISP-5346

\begin{tabular}{|c|c|c|c|}
\hline Region & Type & Most similar known cluster & \\
\hline Region 1 & $\begin{array}{l}\text { Terpene, T1PKS, NRPS-like, phosphonate, nucleo- } \\
\text { side, lanthipeptide }\end{array}$ & Phosphinothricintripeptide & $17.00 \%$ \\
\hline Region 2 & NRPS-like, T1PKS & Miharamycin A/miharamycin B & $40.00 \%$ \\
\hline Region 3 & PKS-like & A-201A & $12.00 \%$ \\
\hline Region 4 & Terpene & & \\
\hline Region 5 & NRPS & Isocomplestatin & $50.00 \%$ \\
\hline Region 6 & Lanthipeptide & AmfS & $80.00 \%$ \\
\hline Region 7 & Lassopeptide & Anantin B1/anantin B2 & $40.00 \%$ \\
\hline Region 8 & Furan, NRPS & Ficellomycin & $3.00 \%$ \\
\hline Region 9 & NRPS-like, NRPS & Friulimicin $\mathrm{A} /$ friulimicin $\mathrm{B} /$ friulimicin $\mathrm{C} /$ friulimicin $\mathrm{D}$ & $27.00 \%$ \\
\hline Region 10 & Amglyccycl & & \\
\hline Region 11 & NRPS & Streptobactin & $47.00 \%$ \\
\hline Region 12 & Amglyccycl & Hygromycin A & $51.00 \%$ \\
\hline Region 13 & Fused, NRPS-like, TfuA-related, NRPS, T1PKS & Thiovarsolin A/thiovarsolin B/thiovarsolin C/thiovarsolin D & $83.00 \%$ \\
\hline Region 14 & NRPS & rimosamide & $42.00 \%$ \\
\hline Region 15 & T2PKS,PKS-like, oligosaccharide, LAP & Dutomycin & $36.00 \%$ \\
\hline Region 16 & Bacteriocin & & \\
\hline Region 17 & Terpene & & \\
\hline Region 18 & hglE-KS, T1PKS & Tetronasin & $9.00 \%$ \\
\hline Region 19 & Lanthipeptide & & \\
\hline Region 20 & Ectoine & Ectoine & $100.00 \%$ \\
\hline Region 21 & NRPS, T2PKS & Kosinostatin & $6.00 \%$ \\
\hline Region 22 & LAP, thiopeptide & Jomthonic acid $\mathrm{A} /$ jomthonic acid $\mathrm{B} /$ jomthonic acid $\mathrm{C}$ & $5.00 \%$ \\
\hline Region 23 & Thiopeptide, LAP & & \\
\hline Region 24 & Terpene & Ansamitocin P-3 & $4.00 \%$ \\
\hline Region 25 & NRPS,CDPS & Purincyclamide & $100.00 \%$ \\
\hline Region 26 & T1PKS & Chlorizidine A & $57.00 \%$ \\
\hline Region 27 & Lanthipeptide & & \\
\hline Region 28 & NRPS, T3PKS & Feglymycin & $42.00 \%$ \\
\hline Region 29 & T1PKS, NRPS, aminocoumarin & Bafilomycin B1 & $83.00 \%$ \\
\hline Region 30 & CDPS & & \\
\hline Region 31 & Terpene & Ebelactone & $5.00 \%$ \\
\hline Region 32 & Siderophore & & \\
\hline Region 33 & Siderophore & Ficellomycin & $3.00 \%$ \\
\hline Region 34 & Siderophore & & \\
\hline Region 35 & Terpene & Hopene & $61.00 \%$ \\
\hline Region 36 & Bacteriocin & & \\
\hline Region 37 & NRPS & & \\
\hline
\end{tabular}

Acknowledgements Open Access funding provided by Projekt DEAL. The bioinformatics support of the BMBF-funded project "BielefeldGießen Center for Microbial Bioinformatics" (BiGi) (Grant 031A533) within the German Network for Bioinformatics Infrastructure (de.NBI) is gratefully acknowledged. This research work has been financed by the German Academic Exchange Service (DAAD) with funds from the German Federal Foreign Office in the frame of the Research Training Network "Novel Cytotoxic Drugs from Extremophilic Actinomycetes" (Project ID 57166072).
Data availability The scaffolds of the whole-genome shotgun project have been deposited in the EMBL database (EBI) under the accession no. OKRJ01000001-OKRJ01000020.

Open Access This article is licensed under a Creative Commons Attribution 4.0 International License, which permits use, sharing, adaptation, distribution and reproduction in any medium or format, as long as you give appropriate credit to the original author(s) and the source, provide a link to the Creative Commons licence, and indicate if changes were made. The images or other third party material in this article are included in the article's Creative Commons licence, unless indicated otherwise in a credit line to the material. If material is not included in 
the article's Creative Commons licence and your intended use is not permitted by statutory regulation or exceeds the permitted use, you will need to obtain permission directly from the copyright holder. To view a copy of this licence, visit http://creativecommons.org/licenses/by/4.0/.

\section{References}

Anderson AS, Wellington EMH (2001) The taxonomy of Streptomyces and related genera. Int J Syst Evol Microbiol 51:797-814

Ayed A, Slama N, Mankai H, Bachkouel S, ElKahoui S, Tabbene O, Limam F (2018) Streptomyces tunisialbus sp. nov., a novel Streptomyces species with antimicrobial activity. Antonie Van Leeuwenhoek 111:1571-1581

Barka EA, Vatsa P, Sanchez L, Gaveau-Vaillant N, Jacquard C, Klenk HP (2016) Taxonomy, physiology, and natural products of Actinobacteria. Microbiol Mol Biol Rev 80:1-43

Bérdy J (2005) Bioactive microbial metabolites. J Antibiot 58:1-26

Bérdy J (2012) Thoughts and facts about antibiotics; where we are now and where we are heading. J Antibiot 65:385-395

Blin K, Wolf T, Chevrette MG, Lu X, Schwalen CJ, Kautsar SA, Suarez Duran HG, de Los Santos ELC, Kim HU, Nave M, Dickschat JS, Mitchell DA, Shelest E, Breitling R, Takano E, Lee SY, Weber T, Medema MH (2017) antiSMASH 4.0-improvements in chemistry prediction and gene cluster boundary identification. Nucleic Acids Res 45(W1):36-41

Blom J, Albaum SP, Doppmeier D, Pühler A, Vorhölter FJ, Zakrzewski M, Goesmann A (2009) EDGAR: a software framework for the comparative analysis of prokaryotic genomes. BMC Bioinform 10:154-159

Blom J, Kreis J, Spänig S, Juhre T, Bertelli C, Ernst C, Goesmann A (2016) EDGAR 2.0: an enhanced software platform for comparative gene content analyses. Nucleic Acids Res 44:22-28

De Lima PRE, Da Silva IR, Martins MK, De Azevedo JL, de Araújo JM (2012) Antibiotics produced by Streptomyces. Braz J Inf Dis 16(5):466-471

Demain A (2014) Importance of microbial natural products and the need to revitalize their discovery. J Ind Microbiol Biotechnol 41:185-201

Goodfellow M (2013) Actinobacterial diversity as a source of new drugs. Microbiologist 14:8-12

Harrison J, Studholme DJ (2014) Recently published Streptomyces genome sequences. Microb Biotechnol 7:373-380

Hopwood DA (2000) Practical Streptomyces genetics, 518. Norwich, England, pp 170-171

Kämpfer P (2012) Genus I. Streptomyces Waksman and Henrici 1943, 339 emend. Witt and Stackebrandt 1990, 370, emend. Wellington,
Stackebrandt, Sanders, Wolstrup and Jorgensen, 1992, 159. In: Goodfellow M, Kämpfer P, Busse H-J, Trujillo M, Suzuki KE, Ludwig W, Whitman WB (eds) Bergey's Manual of Systematic Bacteriology, vol 5, 2nd edn. Springer, New York, pp 1455-1767

Konstantinidis KT, Tiedje JM (2005) Genomic insights that advance the species definition for prokaryotes. Proc Natl Acad Sci USA 102:2567-2572

Lee LH, Chan KG, Stach J, Elizabeth Wellington EMH, Goh BH (2018) Editorial: the search for biological active agent(s) From actinobacteria. Front Microbiol 9:824-829

Meier-Kolthoff JP, Auch AF, Klenk HP, Göker M (2013) Genome sequence-based species delimitation with confidence intervals and improved distance functions. BMC Bioinform 14:60

Meyer F, Goesmann A, McHardy AC, Bartels D, Bekel T, Clausen J, Kalinowski J, Linke B, Rupp O, Giegerich R, Pühler A (2003) $\mathrm{GenDB}$ - an open source genome annotation system for prokaryote genomes. Nucleic Acids Res 31:2187-2195

Molloy EM, Tietz JI, Blair PM, Mitchell DA (2016) Biological characterization of the hygrobafilomycin antibiotic JBIR-100 and bioinformatic insights into the hygrolide family of natural products. Bioorg Med Chem 24:6276-6290

Schatz A, Bugle E, Waksman SA (1944) Streptomycin, a substance exhibiting antibiotic activity against Gram-positive and Gramnegative bacteria. Proc Soc Exp Biol Med 55:66-69

Seemann T (2014) Prokka: rapid prokaryotic genome annotation. Bioinformatics 30:2068-2069

Shirling EB, Gottlieb D (1966) Methods for characterization of Streptomyces species. Int J Syst Bacteriol 16:313-340

Tiwari K, Gupta RK (2012) Rare actinomycetes: a potential storehouse for novel antibiotics. Crit Rev Biotechnol 32:108-132

Waksman SA, Henrici AT (1943) The nomenclature and classification of the actinomycetes. J Bacteriol 46:337-341

Ward AC, Allenby NEE (2018) Genome mining for the search and discovery of bioactive compounds: the Streptomyces paradigm. FEMS Microbiol Lett. https://doi.org/10.1093/femsle/fny240

Yücel O, Wibberg D, Philipp B, Kalinowski J (2017) Genome sequence of the bile salt-degrading bacterium Novosphingobium sp. strain Chol11, a model organism for bacterial steroid catabolism. Genome Announc 6(1):e01372-e1417

Zhang L, Ruan C, Peng F, Deng Z, Hong K (2016) Streptomyces arcticus sp. nov., isolated from frozen soil. Int J Syst Evol Microbiol 66:1482-1487

Publisher's Note Springer Nature remains neutral with regard to jurisdictional claims in published maps and institutional affiliations. 